\title{
On Optimal Tolerancing in Computer-Aided Design
}

\author{
H. Pottmann ${ }^{a}$, B. Odehnal ${ }^{a}$, M. Peternell ${ }^{a}$, J. Wallner ${ }^{a}$, R. Ait Haddou ${ }^{b}$ \\ ${ }^{a}$ Institute of Geometry, Vienna University of Technology, \\ Wiedner Hauptstraße 8-10, A-1040 Wien, Austria \\ ${ }^{b}$ Department of Mechanical Engineering, University of Calgary, \\ 25000 University Dr. NW, Calgary, Alberta, Canada T2N 1N4
}

\begin{abstract}
A geometric approach to the computation of precise or well approximated tolerance zones for CAD constructions is given. We continue a previous study of linear constructions and freeform curve and surface schemes under the assumption of convex tolerance regions for points. The computation of the boundaries of the tolerance zones for curves / surfaces is discussed. We also study congruence transformations in the presence of errors and families of circles arising in metric constructions under the assumption of tolerances in the input. The classical cyclographic mapping as well as ideas from convexity and classical differential geometry appear as central geometric tools.
\end{abstract}

\section{Introduction}

In the past decades, a huge amount of research has been devoted to geometric problems arising in Computer Aided Design. Almost always one assumes precise input or one is not so much concerned about the effect of input errors or tolerances on the output. However, for practical purposes, this is a fundamental question. A closer look exhibits a possible reason for a lack of research in this area: even for simple constructions, the analysis of tolerances and their interdependencies is quite complex.

Before outlining the contributions of the present paper, let us briefly review the state of the art in geometric tolerancing.

Tolerance logics are tables with the values of the dimensions (distances, radii, angles ...) for a special drawing (designed value, minimal/maximal value and mean value). Each drawing requires its own table. One disadvantage is the (semi-)manual creation of the tolerance logics. The designer has to specify the values of possibly hundreds of dimensions, a time consuming and risky process, since only a well trained designer may be able to find the critical tolerance combinations (see [16] and references therein).
An alternative is the stochastic approach, where one assumes a distribution for each dimension. Then, with e.g. a Monte-Carlo simulation one derives an estimation of the minimal/maximal distance of two points on the designed object. Again the designer has to find critical situations. An advantage of the method is a sensitivity analysis which one can derive from the simulation and its applicability to $3 \mathrm{D}$ problems [44].

Worst case approaches with no manual interaction to find critical tolerance situations give the designer an easy to use tool for the tolerance analysis. Requicha [38] introduced such a model based on tolerance zones. Tolerance zones are also a very intuitive visualization technique for error propagation in geometric constructions.

Robust geometric computing using tolerance zones and interval arithmetic has recently received much attention $[1,17,20,18,40,41,42,43]$. Interval arithmetic possesses a lot of computationally attractive features, but it tends to overestimate the error and thus may produce too large tolerance zones.

For 2-dimensional CAD constructions, important progress on worst case tolerancing has been made by C. U. Hinze [16], who analyzed several frequently appearing elementary constructions in the plane involving straight lines and circles. His tolerance zones for points are convex and bounded by segments of straight lines and circles. An application to collision problems involving toleranced objects has been given by M. Aichinger [2].

Recently, J. Wallner et al. [46] proposed to use more general convex tolerance zones for points and showed how linear constructions in arbitrary dimensions and a variety of curve and surface design schemes can be effectively analyzed using ideas from convexity. Moreover, the dramatic change in the level of difficulty when switching to metric constructions has been illustrated by the seemingly simple problem of constructing a circle through three points (in the plane or in space). In fact, this problem is so complex that only the special case of balls for the tolerance regions of the three input points has been analyzed. 
In the present paper, we would like to continue these developments with a major focus on metric constructions and the computation of boundaries of tolerance zones. After briefly reviewing the basic facts on linear constructions and tolerance zones for freeform curves and surfaces, we present a surprisingly simple way of computing curvatures of tolerance zone boundaries for curves in the plane. This allows us to apply high accuracy approximation schemes to represent such boundaries. An extension to surfaces is just outlined and shall be discussed in a separate contribution. Moreover, we study planar displacements in the presence of input errors.

We then introduce to a very useful concept from geometry: the cyclographic mapping. It is applied to the tolerance anlysis of constructions involving circles. Finally, a brief outlook on future research in the wide unexplored area of geometric tolerancing is given.

The present contribution is thought as a presentation of basic results as well as ideas and tools from geometry which simplify the study of tolerance zones in geometric constructions. Many topics we touch need further research. Moreover, in order to get the necessary understanding, we discuss certain constructions in more generality than the actual implementation might exhibit. For example, we currently admit arbitrary convex bodies as tolerance zones for points. Knowing the type and complexity of the arising constructions, we will later decide which restrictions have to be made for a practical realization of our tolerance analysis.

\section{Linear combinations of points and applica- tions to freeform curves and surfaces}

\subsection{Support function and linear combination of convex bodies}

Since we will restrict our study to convex tolerance zones for points, we present a brief outline of necessary facts from convex geometry.

One of the main tools for studying compact convex bodies of $\mathbb{R}^{d}$ is the support function. This concept has been used for a long time and there is large amount of literature, including some monographs on convex geometry (see e.g. [15] for a detailed overview of the whole field of convex geometry).

We call a plane $\varepsilon$ a support plane of a convex body $K$ if $K$ has a point in common with $\varepsilon$ and $K$ is entirely contained in one of the two closed half-spaces defined by $\varepsilon$. For all unit vectors $n$ there is a unique plane $\varepsilon(n)$ orthogonal to $n$ which is a support plane of $K$ such that $K$ lies in the halfspace of $\varepsilon$ which is indicated by $-n$. The oriented distance of this plane to the previously fixed origin is the value of the support function $s(n)$. Then $\varepsilon$ has the equation $x \cdot n=s(n)$ and $K$ lies in the half-space $x \cdot n \leq s(n)$.
The domain of a support function is the unit sphere $S^{d-1}$ of $\mathbb{R}^{d}$. In the plane $(n=2)$ it is sometimes useful to make the domain of the support function the interval $[0,2 \pi]$, where an angle $\phi$ is identified with the appropriate point of the unit circle. We will never do this, because we sometimes evaluate the support function at vectors $n$ and $-n$, which are opposite points of the unit sphere. In the case $n=2$ opposite points correspond to angles $\phi, \phi+\pi$, and $-\phi$ means something different. In order to avoid confusion, let us state explicitly that the minus sign always indicates the opposite point of the unit sphere.

If $K$ is a convex body, $\lambda K$ is the set of all $\lambda x$ with $x \in K$. For two convex bodies $K_{1}, K_{2}$, their Minkowski sum $K=$ $K_{1}+K_{2}$ is defined as the set of all $x_{1}+x_{2}$ with $x_{1} \in K_{1}$, $x_{2} \in K_{2}$.

For real numbers $t_{1}, t_{2}$ we then can define the body $K=t_{1} K_{1}+t_{2} K_{2}$. Especially we define affine combinations $(1-t) K_{1}+t K_{2}$ of convex bodies, and convex combinations, which are affine combinations with $0 \leq t \leq 1$.

Among the basic properties of support functions are the following: If $s: S^{d-1} \rightarrow \mathbb{R}$ is the support function of $K$, then $K^{\prime}=\lambda K$ has the support function $s^{\prime}(n)=\lambda s(n)$ if $\lambda \geq 0$. The support function $s^{\prime}$ of $-K$ is given by $s^{\prime}(n)=s(-n)$. If $s_{1}, s_{2}$ are the support functions of $K_{1}, K_{2}$, resp., and $0 \leq t \leq 1$, then the convex body $K=(1-t) K_{1}+t K_{2}$ has the support function $s=(1-t) s_{1}+t s_{2}$. From this it easily follows that the support function of the convex body $\lambda_{1} K_{1}+\lambda_{2} K_{2}$ equals $\lambda_{1} s_{1}+\lambda_{2} s_{2}$ if both $\lambda_{1}, \lambda_{2}$ are nonnegative.

As an application of this, we derive the support function $s$ of a linear combination $K=\sum \lambda_{i} K_{i}$ of convex bodies $K_{i}$ with support functions $s_{i}$,

$$
K=\sum_{\lambda_{i}>0} \lambda_{i} K_{i}+\sum_{\lambda_{i}<0} \lambda_{i} K_{i}=\sum_{\lambda_{i}>0} \lambda_{i} K_{i}+\sum_{\lambda_{i}<0}\left(-\lambda_{i}\right)\left(-K_{i}\right) .
$$

The support function $s_{i}^{\prime}$ of $-K_{i}$ is given by $s_{i}^{\prime}(n)=s_{i}(-n)$. Clearly, the support function of a positive linear combination of convex bodies is the same linear combination of their support functions. Thus, the convex body $K$ possesses the support function

$$
s(n)=\sum_{\lambda_{i}>0} \lambda_{i} s_{i}(n)+\sum_{\lambda_{i}<0}\left(-\lambda_{i}\right) s_{i}(-n)
$$

We see that Minkowski sums are the basic entity for linear combinations of convex bodies. For computational issues of Minkowski sums in the plane, we refer the reader to Kaul and Farouki [21] and Lee et al. [23]. In the latter paper, Minkowski sums are studied for arbitrary planar domains. This is much more subtle than Minkowski sums of convex bodies, which is one reason for our limitation to convex tolerance zones for points. Instead of the tolerance region of a point we will also speak of a 'fat point'. Likewise, tolerance regions to curves or surfaces will be briefly called 'fat curves/surfaces'. 


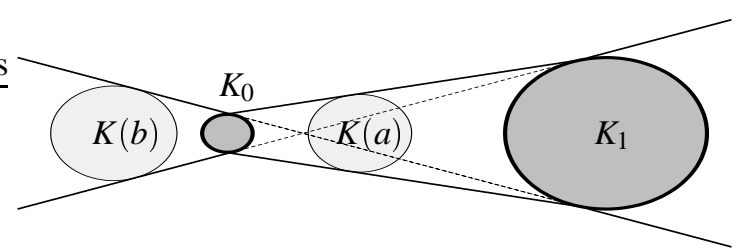

Figure 1. Boundary of a fat line in the plane connecting fat points $K_{0}$ and $K_{1}$ and tolerance regions to affinely combined points $K(a)$ and $K(b)(a=0.35, b=-0.5)$

\subsection{Curves and surfaces defined by control points}

Let us mention some applications of linear combinations of convex bodies to geometric tolerancing. For more details, we refer the reader to Wallner et al. [46].

\section{Spanning line and plane}

Given the convex tolerance regions $K_{0}, K_{1}$ to two points in $\mathbb{R}^{d}$, the tolerance regions for the points on the connecting line are given by

$$
K(t)=(1-t) K_{0}+t K_{1} .
$$

For each $t$, this is a convex domain, and the union of all domains $K(t)$ is the tolerance zone of the connecting line. It is bounded by (part of) the envelope of $K(t)$.

Let us assume disjoint regions $K_{0}, K_{1}$. Then, in the plane, the tolerance region of the connecting line of the two fat points $K_{0}, K_{1}$ is bounded by parts of the four common support lines (tangents for smooth $K_{i}$ ) (see Fig. 1).

In 3-space, the bounding surface of the tolerance region to the spanning line consists of parts of developable surfaces joining the two convex surfaces $K_{1}, K_{2}$. Their computation is briefly discussed below.

Points of the spanning plane of three fat points $K_{i}$ in $\mathbb{R}^{3}$ are associated to the tolerance regions

$$
K(u, v)=u K_{0}+v K_{1}+(1-u-v) K_{2} .
$$

Assuming disjoint regions $K_{i}$, the boundary of the tolerance zone to the plane has eight planar faces, which are parts of the common support planes of the three convex bodies $K_{0}, K_{1}, K_{2}$. This is illustrated in Fig. 2 with three balls $K_{0}, K_{1}, K_{2}$. Note that the points of tangency of the common tangent planes with the spheres are not at the boundary of the tolerance region for the spanning plane.

\section{Developable surfaces joining two curves or surfaces}

Consider two curves or surfaces $c_{1}, c_{2}$. Then, a developable surface joining the two objects is the envelope of all planes

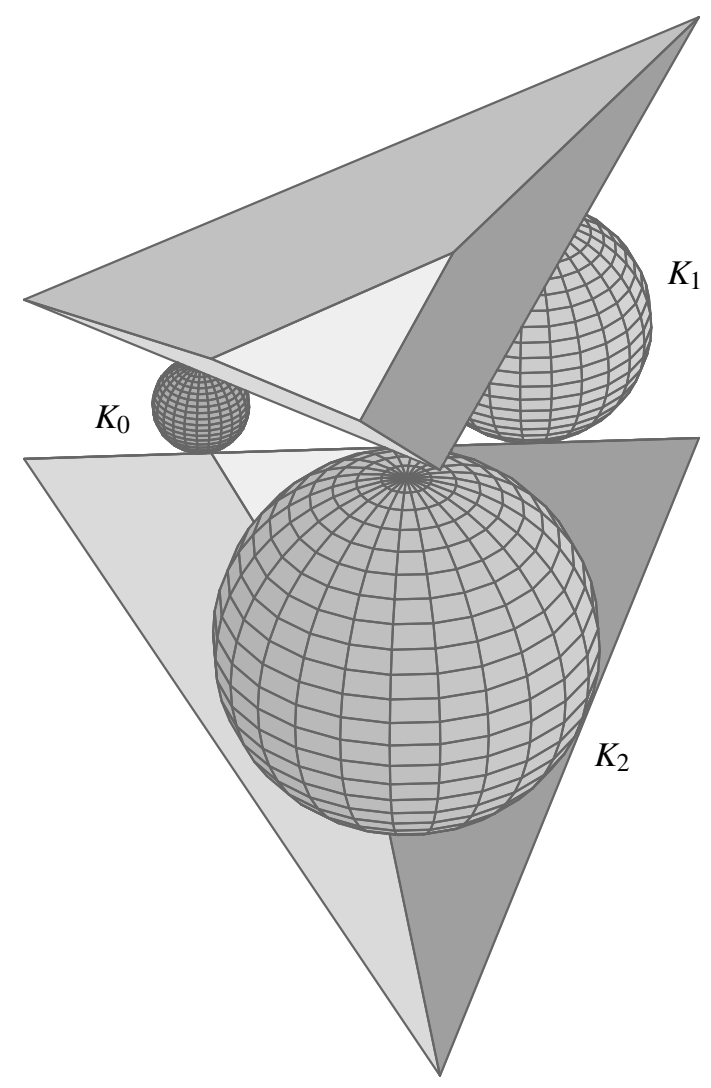

Figure 2. Boundary of the tolerance region for
the spanning plane of three fat points (balls)

which touch both $c_{1}$ and $c_{2}$. Here, a tangent plane to a curve is a plane through a tangent of the curve. A developable surface contains a one-parameter family of straight lines (rulings, generators). The generators are the connections of the contact points $p_{i} \in c_{i}$ of common tangent planes of $c_{1}$ and $c_{2}$ (Fig. 3).

We may view $c_{i}$ as dual objects, i.e., as sets of their tangent planes. The computation of a joining developable surface is therefore dual to the computation of an intersection curve of two surfaces. Just like the intersection curve may have different topological components, the connecting developable may also consist of different components.

Consider two non-intersecting convex bodies $K_{0}, K_{1}$ in $\mathbb{R}^{3}$ with support functions $s_{0}, s_{1}$. We admit piecewise smooth bodies and thus speak of support planes instead of tangent planes. The normals $n$ of common support planes satisfy

$$
s_{0}(n)-s_{1}(n)=0
$$

if $K_{0}$ and $K_{1}$ lie on the same side of the support plane. Normals to common support planes, where $K_{0}$ and $K_{1}$ lie on 


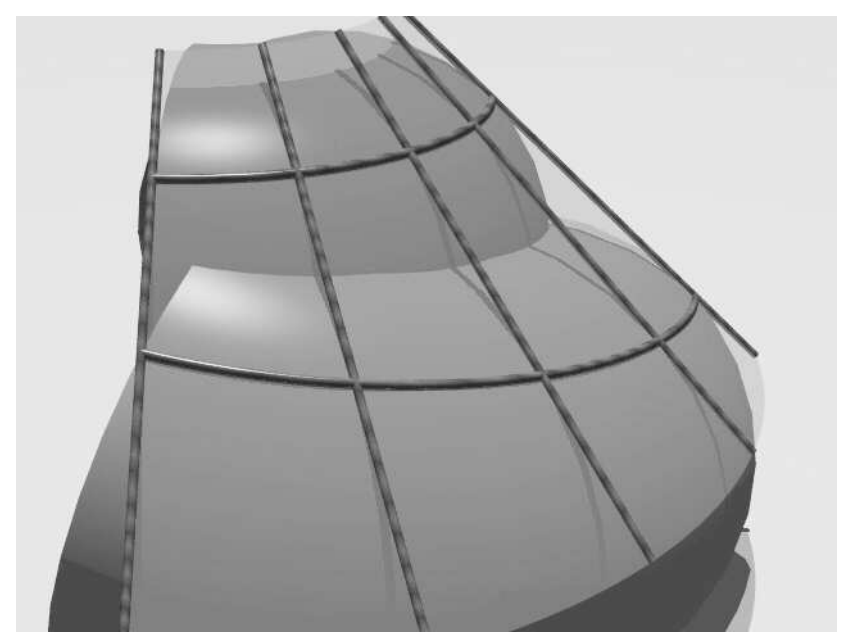

Figure 3. Connecting developable surface of two surfaces

different sides, are characterized by

$$
s_{0}(n)+s_{1}(-n)=0 .
$$

Equation (2) belongs to the first component of the joining developable. Its part between $K_{0}$ and $K_{1}$ lies in the boundary of the tolerance zone for the line segment $(1-t) K_{0}+$ $t K_{1}, t \in[0,1]$. Equation (3) is describing the normals of the developable surface, which contributes to the boundary of the tolerance zone for $t<0$ and $t>1$.

The normals satisfying (2) or (3) form curves on the unit sphere. These are the Gaussian images of the two components of the joining developable surface. Algorithmically, we have to compute the zero set of a function on the unit sphere. In general, parameterizations of these spherical curves, say $k_{1}(t)$ and $k_{2}(t)$, can be found only by approximation algorithms. The developable surfaces are then enveloped by planes $x \cdot k_{i}(t)=s_{0}\left(k_{i}(t)\right)$ for $i=1,2$. Then, algorithms for working with this dual representation may be employed. For the latter topic and approximation with rational developable surfaces, see e.g. [35, 37]. In the present application, simple boundaries such as those composed by cones of revolution are an advantage [24, 25].

$K_{0}$ and $K_{1}$ may degenerate to planar convex domains. Such a case is illustrated in Figure 4, where $K_{i}$ lie in parallel planes, say $x_{3}=0$ and $x_{3}=1$. Just the component to equation (2) between $K_{0}$ and $K_{1}\left(0 \leq x_{3} \leq 1\right)$ is displayed. A plane $x_{3}=t, t \in[0,1]$ cuts the developable surface in the boundary of $K(t)$. In a parallel projection onto $x_{3}=0$ we see the planar figure of a set of convex domains $K(t)=(1-t) K_{0}+t K_{1}$.

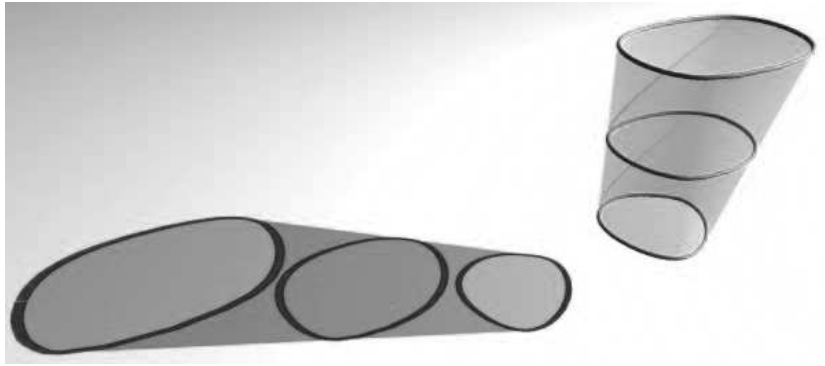

Figure 4. Developable surface and convex combination of convex domains

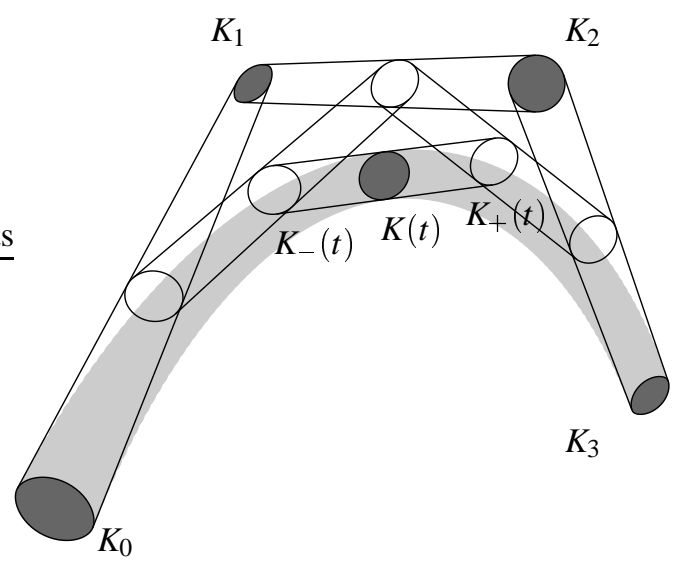

Figure 5. Tolerance zone to Bézier curve with $0 \leq t \leq 1$

\section{Tolerance regions of Bézier curves}

A large amount of curve and surface schemes in Computer Aided Geometric Design consists, in principle, of appropriate linear combinations of control points $b_{i}$. This allows us to apply the same methods as above to the tolerance analysis for many freeform curves and surfaces [46].

Let us look at the example of Bézier curves.

A control point $b_{i}$ of a Bézier curve corresponds to a convex body $K_{i}$ as its tolerance zone. It shall have support function $s_{i}$. The tolerance zone for the curve point with parameter value $t$ is the convex body

$$
K(t)=\sum_{i=0}^{n} B_{i}^{n}(t) K_{i} .
$$

The sign of the ordinary Bernstein polynomials is given by

$$
\operatorname{sgn}\left(B_{i}^{n}(t)\right)=\operatorname{sgn}(t)^{n-i} \operatorname{sgn}(1-t)^{i}
$$

and so the support function $s$ of $K(t)$ is given by

$$
s(n)=\sum B_{i}^{n}(t) s_{i}^{\star}(n),
$$




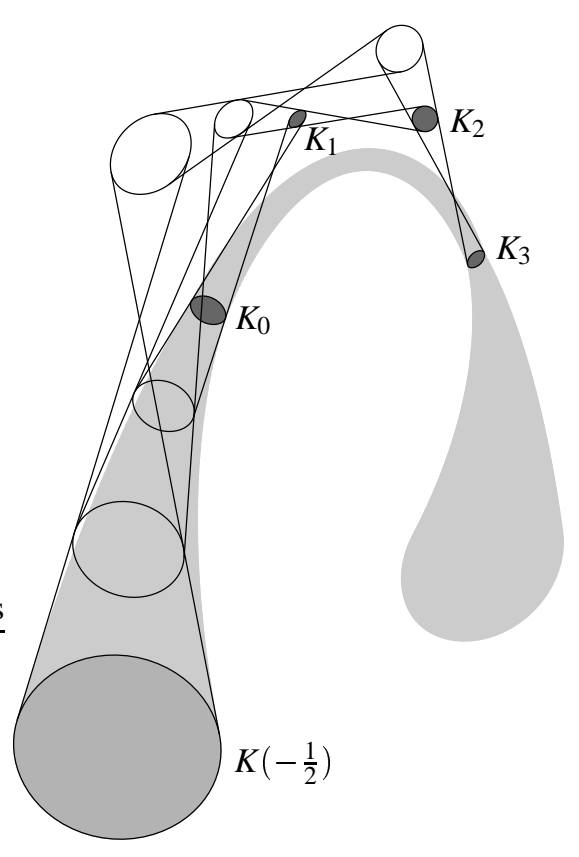

Figure 6. Tolerance zone to Bézier curve with $-1 / 2 \leq t \leq 3 / 2$

where

$s_{i}^{\star}(n)= \begin{cases}s_{i}(n) \text { for } \quad t<0, n-i \text { even, or } \\ & t>1, i \text { even, or } \\ & 0 \leq t \leq 1 \\ -s_{i}(-n) \quad & t<0, n-i \text { odd, or } \quad t>1, i \text { odd. }\end{cases}$

This can be symbolized by the following diagram:

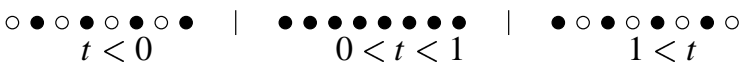

Here, the dots are symbolizing the signs of the basis functions $B_{0}^{n}, \ldots, B_{n}^{n}$ ( $n=7$ in our example), and fat points indicate a positive sign.

A figure of the union of all possible $K$ 's when $t$ ranges in an interval, can be seen in Fig. 5 and Fig. 6. Fig. 5 also illustrates the computation of points and tangents of the boundary of the tolerance zone: In the plane, it is based on common tangents to the tolerance regions $K_{-}(t), K_{+}(t)$ which arise in the de Casteljau algorithm. In $\mathbb{R}^{3}$, the curve, along which the region $K(t)$ touches the envelope ('characteristic curve') lies on the joining developable surface of $K_{-}(t), K_{+}(t)$ (see connecting line of two fat points). From Fig. 6 it is clearly seen that in the case $t \notin[0,1]$, the size of $K(t)$ is rapidly increasing with the distance of $t$ to the standard interval $[0,1]$. So the often cited 'optimality' of the Bernstein basis is again seen to be valid only in the interval $[0,1]$ (see [11]).

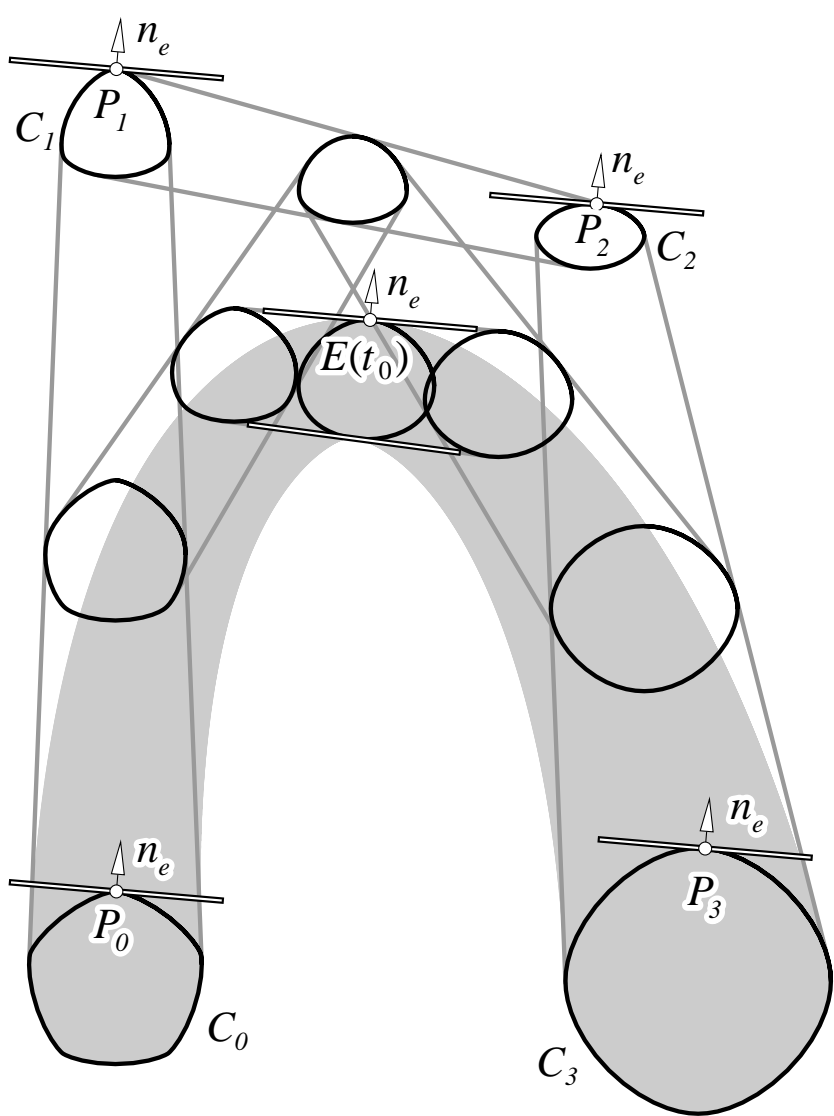

\section{Figure 7. To the computation of the curvature of the boundary of a fat Bezier curve at a point $E\left(t_{0}\right)$}

More details on the computation of the tolerance zone boundaries are found in [46], but there are still open questions in this area. One will be addressed in the following subsection.

\section{Curvature of tolerance zone boundaries}

Let us first focus on a fat Bézier curve in the plane and discuss the computation of its boundary. Apart from special cases discussed below, closed form expressions of the boundary will not be obtained since the problem of computing common tangents of the fat points $K_{-}, K_{+}$in the de Casteljau construction will in general not have a closed form solution. Hence we are interested in a good approximation of the boundary, for which it is desirable to know the curvatures at discrete boundary points. Then, appropriate curve approximation schemes such as high accuracy geometric Hermite interpolation with polynomial cubics [8] or osculating arc splines [28] may be applied.

We consider a fat Bézier curve of degree $n$, whose fat 
control points $K_{i}$ shall have $C^{2}$ boundary curves denoted by $C_{i}$. For convenience, we limit ourselves to the parameter interval $[0,1]$; extensions follow immediately from the discussions above. The one-parameter family of fat curve points $K(t)$ has convex $C^{2}$ boundary curves $C(t)$, whose envelope $E$ (eventually after trimming away parts in case of self-intersections of $E$ ) forms, together with parts of $C(0)$ and $C(1)$ the boundary of the fat Bézier curve (see Fig. 5).

We are interested in the curvature of $E$ at an envelope point $E\left(t_{0}\right) \in C\left(t_{0}\right)$. Let $n_{e}$ be the outward unit normal of $E$ and $C\left(t_{0}\right)$ at $E\left(t_{0}\right)$ and consider those points $P_{i}$ on the control domain boundaries $C_{i}$, which also possess the outward unit normal $n_{e}$ (see Fig. 7). Points $P_{i}, i=0, \ldots, n$ are considered as control points of a Bézier curve $B$. It follows immediately from (4), that the curve point $B(t)$ is exactly that point of the tolerance zone boundary $C(t)$ whose unit normal is $n_{e}$ (for all $t \in[0,1]$ ). Moreover, since the curvature radius of a convex curve is the sum of the support function and its second derivative, we see that the curvature radius $\rho_{c}$ of $C\left(t_{0}\right)$ at $E\left(t_{0}\right)$ is computed from the curvature radii $\rho_{i}$ of $C_{i}$ at $P_{i}$ by

$$
\rho_{c}=\sum_{i=0}^{n} B_{i}^{n}\left(t_{0}\right) \rho_{i}
$$

With the curvature radius $\rho_{b}$ of the Bézier curve $B$ at $B\left(t_{0}\right)=$ $E\left(t_{0}\right)$, we are then able to compute the curvature radius $\rho_{e}$ of the envelope at $E(t)$ :

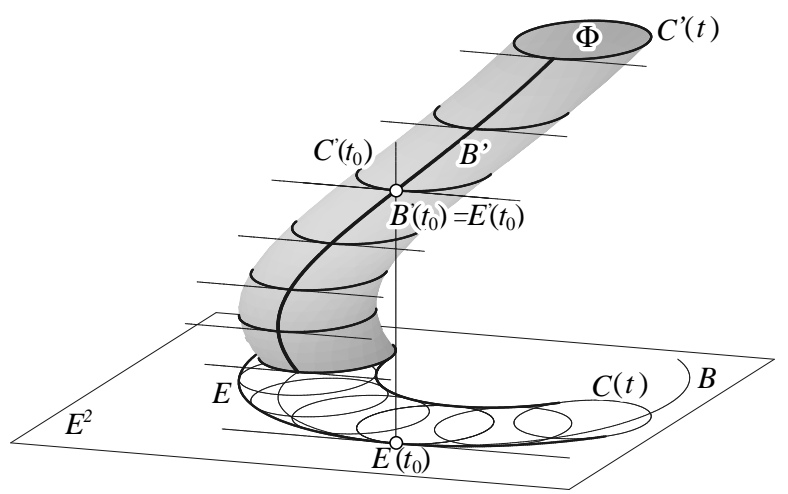

Figure 8. Spatial interpretation of curvature construction of zone boundaries

Theorem 1. The curvature radius $\rho_{e}$ of the boundary of a fat Bézier curve at a point $E\left(t_{0}\right)$ with normal $n_{e}$ is computed as follows. Compute the curvature radii $\rho_{i}$ of the control domain boundaries $C_{i}$ at points $P_{i}$ with normal $n_{e}$, and compute the curvature radius $\rho_{b}$ of the Bézier curve with control points $P_{i}$ at the curve point $E\left(t_{0}\right)$ to parameter $t_{0}$. Then,

$$
\rho_{e}=\rho_{b}+\sum_{i=0}^{n} B_{i}^{n}\left(t_{0}\right) \rho_{i} .
$$

All curvature radii are the inverse curvatures, i.e., taken with sign.

Proof: Our proof is based on an interpretation of the planar figure as projection of a spatial one. We translate each curve $C(t)$ by the vector $(0,0, t)$ up to height $z=t$ in 3 -space, see Figure 8 . The translated domain boundaries, denoted by $C^{\prime}(t)$, form the contour curves $z=t=$ const of a surface $\Phi$ in $\mathbb{R}^{3}$. The silhouette of $\Phi$ for orthogonal projection into $z=0$ is the envelope $E$. The lifted points $B^{\prime}(t) \in C^{\prime}(t)$ of the auxiliary Bézier curve $B$ form a Bézier curve $B^{\prime}$ on the surface $\Phi$. The tangents to the level curves $C^{\prime}(t)$ at its points $B^{\prime}(t)$ are parallel and form a general cylinder surface which touches $\Phi$ along the curve $B^{\prime}$. Hence, the tangents of $B^{\prime}$ are conjugate (in the sense of differential geometry) to the horizontal cylinder generators. In particular, at the lifted envelope point $E^{\prime}\left(t_{0}\right)$ (point on the contour of $\Phi$ for $z$-parallel projection), the tangent to the level curve $C^{\prime}\left(t_{0}\right)$ and the tangent to the Bézier curve $B^{\prime}$ are conjugate. By a theorem of H. Schaal on the curvature radius of the silhouette of a surface $[39,45]$, we conclude that the sum of the curvature radii of the projections $C\left(t_{0}\right), B$ of the two curves at the silhouette point $E\left(t_{0}\right)$ equals the curvature radius $\rho_{e}$ of the silhouette. With (5), this proves the claim of the theorem.

It is obvious that an analogous theorem holds for other linear curve schemes (B-splines, interpolatory $C^{2}$ cubic splines,...) as well. The result can also be generalized to surfaces. Here, curvature radii (inverse curvatures) are replaced by inverse Weingarten maps of surfaces which characterize the curvature behaviour. The derivation of this result is beyond the scope of the present paper and shall be given elsewhere. We just mention that a special case concerns curvature computation for general offset surfaces [34].

Remark. The limitation to $C^{2}$ boundaries $C_{i}$ of the fat control points is not necessary. Replacing normals by normals to support lines, we can compute points $P_{i} \in C_{i}$. If, at a point $P_{i}$ curve $C_{i}$ has a tangent discontinuity (and $n_{e}$ is not normal to a boundary curve segment ending there), we assign curvature radius 0 to it. If $n_{e}$ is normal at $P_{i}$ and there is a curvature discontinuity of $C_{i}$, we compute with right and left limit of the curvature and obtain right and left limit curvature for the envelope.

\section{'Interval' curves and surfaces}

If the coordinates of control points are known to lie in certain intervals, then an equivalent formulation of this is that the control point itself may vary in some orthogonal parallelotope whose faces are parallel to the coordinate hyperplanes. In the plane, interval bounds on the coordinates of points are equivalent to working with tolerance zones of 
points, which are rectangles with edges parallel to the coordinate axes.

The procedure of linear combination, especially of convex combination of such parallelotopes again produces convex orthogonal parallelotopes. If the linear combination is with nonnegative factors, then the coordinates of the faces of the box containing the eventual curve or surface point can be computed by the same linear combination. Interval Bézier curves are studied in [40]. Interval B-splines and their applications to curves and surfaces were studied in $[17,20,41,42,43]$. There, the authors also address the boundary computation in the plane, which is simpler than above, since the boundary consists of special Bézier or Bspline curves and straight line segments parallel to the coordinate axes. It may be a good exercise to verify this with help of the spatial interpretation given above.

\section{Ball curves and surfaces}

Another class of convex bodies closed under the operation of linear combination are the Euclidean balls of radius $r$, which in dimension one coincide with the intervals, and which in dimension two are usually called disks. If the linear combination of control points which yields the eventual curve/surface point is nonnegative, then both the midpoint and the radius of the eventual balls is obtained by the same linear combination of the midpoints and radii of the control point balls. Planar 'disk' Bézier curves are studied in [26]. In dimensions 2 and 3, ball Bézier curves are cyclographic images of Bézier curves $C^{\prime}$ in $\mathbb{R}^{3}$ and $\mathbb{R}^{4}$, respectively (see [36] and the following section). We should also remark that the boundary of the domain to a planar disk Bézier curve can be parameterized using polynomials and square roots; it is in general not a rational curve. Only for special curves $C^{\prime}$ in $\mathbb{R}^{3}$, which are a counterpart to the Pythagoreanhodograph $(\mathrm{PH})$ curves of Farouki and Sakkalis [14, 10], these envelopes are rational [29]. Surprisingly, canal surfaces which appear as boundaries of ball Bézier curves in $\mathbb{R}^{3}$ always possess a rational parameterization [32].

\section{General offsets}

A further simple special case occurs if all control domains are translates of a single convex domain $D$. Then, all fat points on the fat curve/surface are translates of $D$.

In case that $D$ is a ball of radius $r$, the fat curve/surface is bounded by the two-sided offset at distance $r$ to the center curve/surface. In the planar curve case, all auxiliary Bézier curves $B$ are translates of the center curve. Hence, we get the well-known result that the curvature radius of an offset is the sum of the curvature radii of the progenitor curve and the offset distance. Offsets, in particular questions on their rationality, have been a very active research area in the past (see e.g. [10, 27, 33]).
Analogously, an arbitrary centrally symmetric convex domain $D$ gives rise to general offsets as boundaries. The curvature formula, even for surfaces, is known in this case [34]. As for classical offsets, it can be formulated with center curve/surface and $D$ only. Rationality of general offsets has also been discussed recently $([3,4,34])$.

\section{Constructions with linear schemes}

So far we are able to compute tolerance regions for linear curve and surface schemes including the special cases of spanning line and plane of points. If a construction involves just these basic elements and intersection operations, the intersections of the tolerance domains have to be computed. This is, particularly in $\mathbb{R}^{3}$, computationally expensive. Moreover, the resulting tolerance regions to intersection points need not be convex. We then replace them by their convex hull. Limitation to special convex domains as tolerance regions for points (such as the 2D approach in [16]) may help to speed up such computations. However, note that we are interested in sharp enclosures of tolerance zones and thus need restrictions that still allow us to get good approximations. This will be a topic for future research.

\subsection{Displacements in the Euclidean plane}

A toleranced translation in the plane may be given by translation vectors $v$ which describe some convex body $V$. Then, the image of a fat point $K$ under the translation is the Minkowski sum $K+V$.

Let us now study a toleranced rotation. We merge Cartesian coordinates $\left(x_{1}, x_{2}\right)$ of points in the plane into complex numbers $x=x_{1}+i x_{2}$. In the complex notation, a rotation around a point $c$ with angle $\phi$ maps $x$ to $x^{\prime}$ via

$$
x^{\prime}=c+(x-c) e^{i \phi}=c\left(1-e^{i \phi}\right)+x e^{i \phi} .
$$

We now assume that the angle $\phi$ is precise, but the center $c$ and point $x$ are fat points (convex domains). Writing the complex number $a=1-e^{i \phi}$ in the form $a=\rho e^{i \psi}$, we see that $c\left(1-e^{i \phi}\right)=c a$ describes a convex domain, obtained from $c$ by a rotation around the origin with angle $\psi$ and a scaling with factor $\rho$. Clearly, $x e^{i \phi}$ is a rotated copy of $x$. Thus, we find the following result: The fat image point is a convex domain, obtained as Minkowski sum of a rotated copy of the fat preimage point and a rotated and scaled copy of the fat rotation center.

In case that the rotation angle $\phi$ is also subject to tolerances, say $\phi$ in some interval $I$, we can use recent work by Farouki, Moon and Ravani $[13,12]$ to study the fat image points. These are in general no longer convex. In order to stay within our approach, we have to form the convex hull of the image domains. 


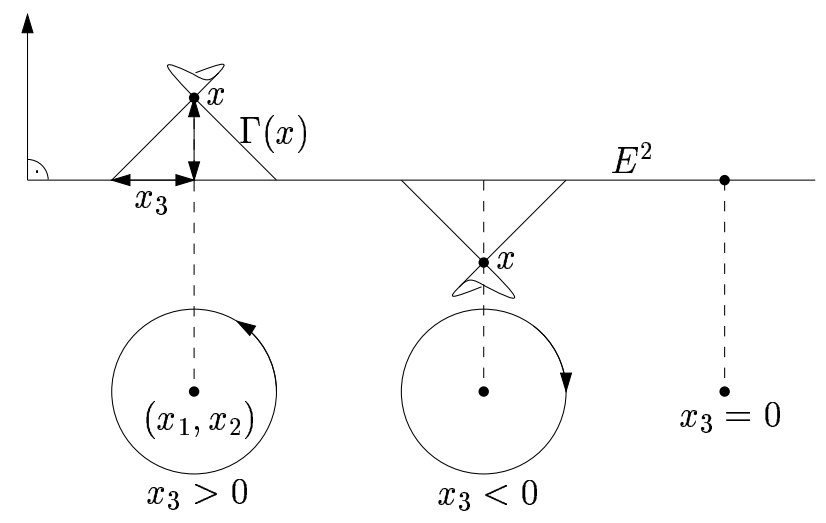

Figure 9. Cyclographic mapping, top and front view

\section{The cyclographic mapping}

In a tolerance analysis of planar metric constructions, tolerance zones for circles play a central role. These are formed by $k$-parameter $(k=1,2,3)$ sets of circles. In this section, we will briefly introduce to a concept from classical geometry, the so-called cyclographic mapping, which is very helpful both for understanding circle constructions and for computing tolerance zones. For a detailed exposition of this interesting classical topic we refer to the monographs by Coolidge [6] and Müller and Krames [31]. In connection with different applications in CAGD, the cyclographic mapping has recently been applied by several authors $[22,33,36]$.

The cyclographic mapping $\zeta$ maps a point $x=\left(x_{1}, x_{2}, x_{3}\right)$ in $\mathbb{R}^{3}$ to an oriented circle in the plane: this circle $c=\zeta(x)$ has midpoint $\left(x_{1}, x_{2}\right)$ and radius $x_{3}$. The sign of $x_{3}$ determines the orientation of the circle (Fig. 9); $x_{3}=0$ characterizes a point in the plane. The image circle of $x$ can also be obtained as follows: consider a cone of revolution with vertex $x$, whose axis is parallel to the $x_{3}$-axis and whose generators form an angle $\gamma=\pi / 4$ with the axis (and the reference plane $E^{2}: x_{3}=0$ ). In the following, we will call such a cone a $\gamma$-cone. Then, $c$ is the intersection of the plane $E^{2}$ and the $\gamma$-cone $\Gamma(x)$ with vertex $x$. This is geometrically a type of projection, but with help of a cone instead of a single projection line.

The orientation of a circle $c$ induces orientations of its tangents; we say that $c$ is in oriented contact with its tangents. Given an oriented line $L \subset E^{2}$, we may ask for all oriented circles $c$ touching $L$. Their cyclographic preimages are the points of a plane $\varepsilon$ through $L$, whose inclination angle against $E^{2}$ is also $\gamma=\pi / 4$ (Fig. 10). Such a plane shall be called a $\gamma$-plane. Hence, we may say that corresponding to oriented lines in $E^{2}$ we have $\gamma$-planes in $\mathbb{R}^{3}$. Oriented

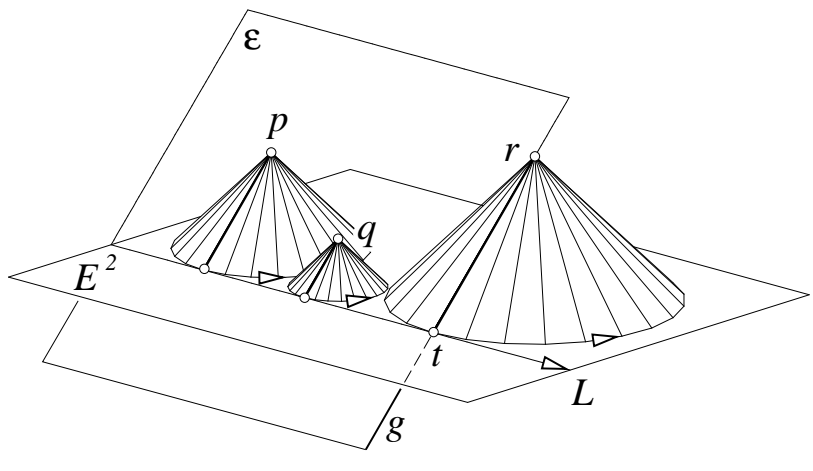

Figure 10. $\gamma$-cones tangent to a $\gamma$-plane

contact between circle and line in the plane is seen as incidence between point and $\gamma$-plane in the spatial model, which is called cyclographic model. All oriented circles touching an oriented line $L$ at a given point $t$ form a one-parameter family. The cyclographic preimage is a ruling $g$ of a $\gamma$-cone, which we call a $\gamma$-line.

Consider an oriented circle $c_{1}$, which is cyclographic image of a point $p_{1}: c_{1}=\zeta\left(p_{1}\right)$. Then, the points of the $\gamma$-cone $\Gamma\left(p_{1}\right)$ are exactly those which are mapped to oriented circles $c \subset E^{2}$ that touch $c_{1}$. Hence, the construction of oriented circles touching three circles $c_{i}, i=1,2,3$ amounts to computing the intersection of the three corresponding $\gamma$-cones $\Gamma\left(p_{i}\right) ; \zeta\left(p_{i}\right)=c_{i}$. This leads to (algebraically counted) two solutions and also solves the Apollonian problem of constructing all circles which are tangent to three given circles. Since there are four essentially different orientation combinations of the three input circles, the Apollonian problem has (algebraically) eight solutions. This spatial approach to the solution of the Apollonian problem ([7]) has been the starting point for studying the cyclographic mapping.

We should mention why three $\gamma$-cones (surfaces of order 2 ), intersect in just two (finite) points, although a careless application of Bezout's theorem might yield $2 \cdot 2 \cdot 2=8$ solutions. This is so, since any two $\gamma$-cones share a conic $\Omega$ at infinity (the cones are translates of each other!). This ideal conic $\Omega$ plays an important role for projective and algebraic considerations.

For our application, orientations are in most cases not necessary. Then, we can either admit only nonnegative radii (confine to the positive half-space $x_{3} \geq 0$ in the spatial model) or work with both orientations (associate a pair of points $\left(m_{1}, m_{2}, \pm r\right)$ to a circle in $\left.E^{2}\right)$. 


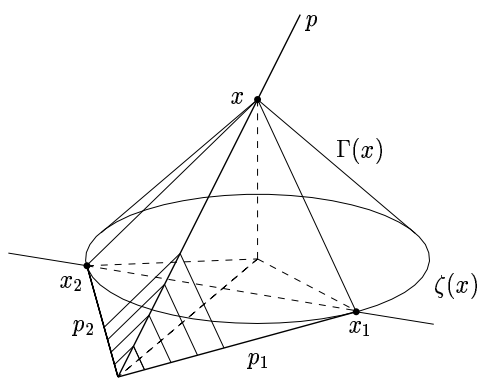

Figure 11. Cyclographic image of a straight line

\subsection{One-parameter sets of circles}

A one-parameter set of circles in $E^{2}$ corresponds to a curve $p$ in the cyclographic model. We are interested in the domain $D(p)$ of the plane which is swept by the image circles $\zeta(x)$ for all points $x \in p$. We call the envelope of the circles $\zeta(x)$ the 'cyclographic image' of the curve $p$ and denote it by $c(p)$.

Let us start with the simplest example, i.e., a straight line $p$. If the line $p$ is inclined under an angle $\alpha<\pi / 4$ against $E^{2}$, the $\gamma$-cones $\Gamma(x)$ for $x \in p$ possess two common tangent planes $\varepsilon_{1}, \varepsilon_{2}$ as envelope, namely the two $\gamma$-planes through $p$. The two planes $\varepsilon_{i}$ intersect $E^{2}$ in two lines $p_{1}, p_{2}$, which bound the domain $D(p)$ traced out by the circles $\zeta(x), x \in p$ (Fig. 11). In connection with an interpretation from special relativity, the current type of lines is called 'space-like'.

If $p$ is a $\gamma$-line ('light line'), we have a unique $\gamma$-plane $\varepsilon$ through $p$ and all image circles touch $\varepsilon \cap E^{2}$ at $p \cap E^{2}$. Here and in the case of a line $p$ with inclination angle $\alpha>\pi / 4$ ('time-like' line, where we have no real envelope), the image circles $\zeta(x), x \in p$, cover the entire plane $E^{2}$. If we restrict to a segment $p$ with end points $a, b$ on such a line, then the domain $D(p)$ is bounded by the image circles $\zeta(a), \zeta(b)$ of the end points.

For an arbitrary regular $C^{1}$ curve $p$, we pick a point $x$ on it and consider its tangent $t$. Since the envelope computation requires first order derivatives only and $t$ is a first order approximant to $p$ at $x$, the following construction yields the contact points $x_{1}, x_{2}$ of $\zeta(x)$ with the envelope (Fig. 12): compute the intersection point $T=t \cap E^{2}$ and pass the tangents $t_{1}, t_{2}$ through $T$ to the image circle $\zeta(x)$; their contact points $x_{1}, x_{2}$ are the envelope points, the tangents $t_{1}, t_{2}$ are tangent to the envelope. The lines $x x_{1}$ and $x x_{2}$ may be considered as rulings of a developable surface through the curve $p$ whose rulings have inclination angle $\gamma=\pi / 4$. Such a $\gamma$-developable can also be viewed as developable surface joining $p$ and the ideal conic $\Omega$.

The domain $D(p)$ is bounded by (parts of) of the following curves: the envelopes to segments of $p$ with space-

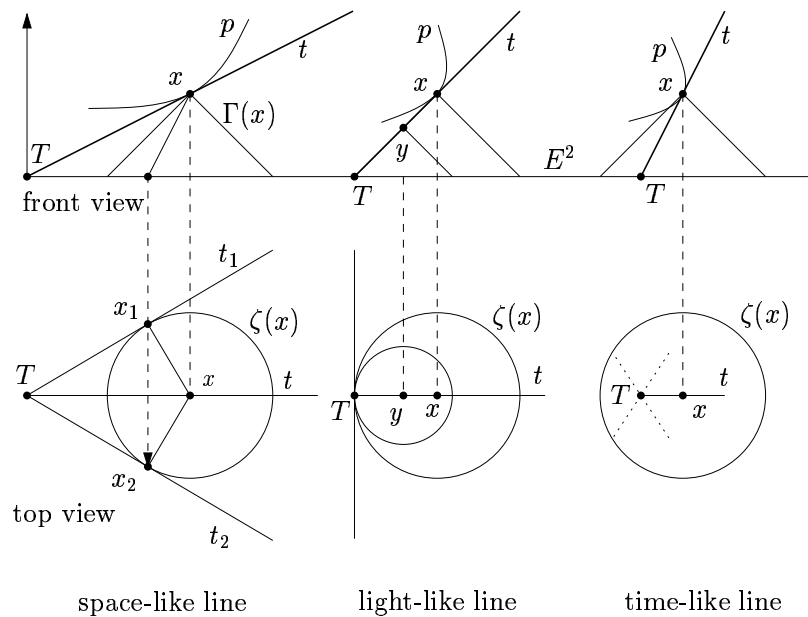

Figure 12. Cyclographic image of point plus
tangent

like tangents and the image circles of the end points of $p$. For piecewise $C^{1}$ curves, we also have to consider the image circles of points with tangent discontinuities. Note that trimming of these curves may be necessary.

It might be surprising that curve segments $p$, all whose tangents are $\gamma$-lines, are not considered for the boundary computation: the tangent surface of such a curve $p$ is a $\gamma$ developable which intersects $E^{2}$ in the envelope curve $c(p)$. The image circles $\zeta(x)$ are osculating circles of the envelope and hence contained in each other (since we require a regular curve $p$ ). Thus, we just have to map the end points of these curve segments in order to get the corresponding domain boundary in $E^{2}$.

For more details on the cyclographic mapping of curves, both from a differential geometric and an algebraic point of view, we refer to the literature $[6,31,36]$.

Example 3.1. An application of the cyclographic mapping of curves are disc Bézier curves in the plane. The tolerance region boundaries for the control points are circles (say with positive radius), which determine points $p_{i}, i=0, \ldots, n$ in the cyclographic model. The spatial Bézier curve segment $p$ with control points $p_{i}$ and parameterized over the standard interval $[0,1]$ has a cyclographic image curve which eventually after trimming - forms together with parts of the end circles $\zeta\left(p_{0}\right)$ and $\zeta\left(p_{n}\right)$ the boundary of the tolerance zone $D(p)$ to the planar Bézier curve segment.

Remark. We pointed out that trimming of the envelope $c(p)$ may be necessary. This occurs in case of selfintersections of $c(p)$ or intersections of different components of $c(p)$. There are 'local' types of self-intersections arising in the neighborhood of typically two cusps of the 


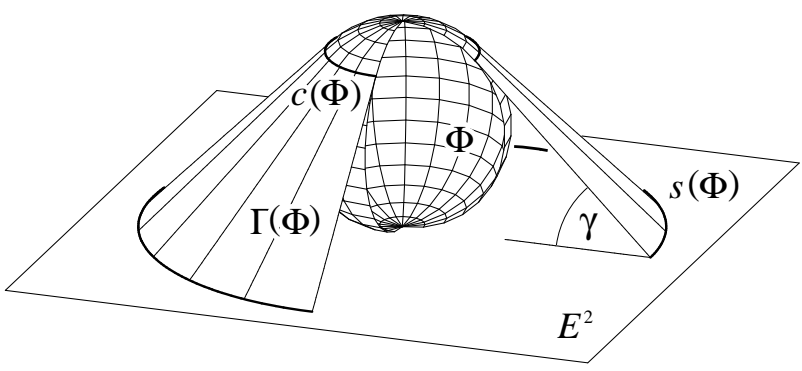

Figure 13. Cyclographic contour and silhouette of a surface (outer part)

envelope (swallow-tail). Those cannot occur if there are no singularities of the envelope; conditions for an envelope without singularities may be found with curvature formula (6). 'Global' types of self-intersections are harder to detect, but under certain global shape conditions it may be possible to prove results which help to speed up tests for selfintersections. Such results could be derived recently in case of offsets, e.g., cyclographic images of curves $p$ in 'horizontal' planes $x_{3}=$ const [47]. Trimming can also be caused by intersections of different parts of the envelope, e.g., those to different segments of the curve $p$, which are separated by segments with time-like tangents.

\subsection{Two-parameter sets of circles}

A two-parameter set of circles is represented by a surface $\Phi$ in the cyclographical model. We are interested in the planar domain $D(\Phi)$ traced out by the circles $\zeta(x), x \in \Phi$, of the system.

Contributions to the boundary of $D$ may come from the cyclographic image of the boundary of the surface $\Phi$ or from the cyclographic silhouette of the surface. The first issue has been discussed already, and thus we are now focusing on the cyclographic silhouette. Similar to the definition of contour and silhouette of a surface with respect to a central or parallel projection, we use the following definition. The cyclographic contour $c(\Phi)$ is the set of all points of the surface $\Phi \in \mathbb{R}^{3}$, whose tangent plane is a $\gamma$-plane. If such points exist, the generic case is the one in which these points form a curve $c$ on the surface (Fig. 13). Since the tangent planes along $c$ form the constant angle $\pi / 4$ with the $x_{3}$-direction, the contour is also an isophote for illumination parallel to the $x_{3}$-axis. The tangent planes along $c$ possess as envelope a $\gamma$-developable $\Gamma(\Phi)$, which may be viewed as connecting developable surface of $\Phi$ and the ideal conic $\Omega$. The intersection curve of $\Gamma(\Phi)$ with the reference plane $E^{2}$ is the cyclographic silhouette $s(\Phi)$ of the surface $\Phi$.

Looking at the cyclographic image of a plane, we see that only a $\gamma$-plane results in a 2-parameter set of circles with an

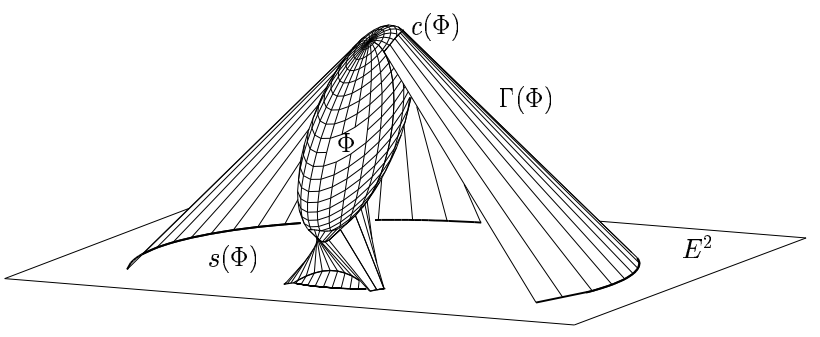

Figure 14. $\gamma$-developable tangent to an ellipsoid

envelope (the intersection of the plane with $E^{2}$ ). From this it follows immediately that a nontrivial envelope of the circle system (i.e., one which does not come from the boundary of $\Phi)$ can only stem from the cyclographic contour, i.e., it must be the cyclographic silhouette.

For trimming the boundary $D(\Phi)$ the following simple fact is helpful: The cyclographic image of parts of the boundary curve $b$ of $\Phi$ can contribute to $D(\Phi)$ only if the tangent planes at its points have an inclination angle less or equal $\pi / 4$ against $E^{2}$.

Example 3.2. The simplest nontrivial surfaces which may be used to approximate a smooth surface $\Phi$ are quadrics. Hence, it is interesting to discuss the cyclographic contour and silhouette of a quadric $\Phi$. We have to construct the connecting developable surface $\Gamma(\Phi)$ between $\Phi$ (surface of algebraic class 2) and the conic at infinity $\Omega$ (as a set of tangent planes, also a surface of class 2 ; it is dual to the point set of a quadratic cone). Hence, the connecting developable is dual to the intersection curve of two surfaces of order 2 and hence it is algebraic (in general not rational) and has class four. This means that, algebraically counted, four tangent planes pass through a general point. Hence, the intersection of $\Gamma(\Phi)$ with the plane $E^{2}$, i.e., the cyclographic silhouette is an algebraic curve of class 4 . The class may be less in case of degree reductions. We do not go into such details here, but mention that this study is dual to degree reductions of central projections of the intersection curve of two quadrics. The developable surface $\Gamma(\Phi)$ possesses $\Omega$ as a self-intersection curve. In general, there are three further self-intersections, which are conics. For a quadric with midpoint $m$, these conics lie in planes through $m$. Clearly, the silhouettes are therefore also cyclographic images of conics. For more details on the silhouettes, which are known in classical geometry as hypercycles, see W. Blaschke [5].

Figure 14 illustrates the case of an ellipsoid. In this example we have two topological components of the boundary of $D(\Phi)$ belonging to the two topological components of the $\gamma$-developable $\Gamma(\Phi)$. However, the (untrimmed) boundary is just one irreducible algebraic curve and the $\gamma$-developable 


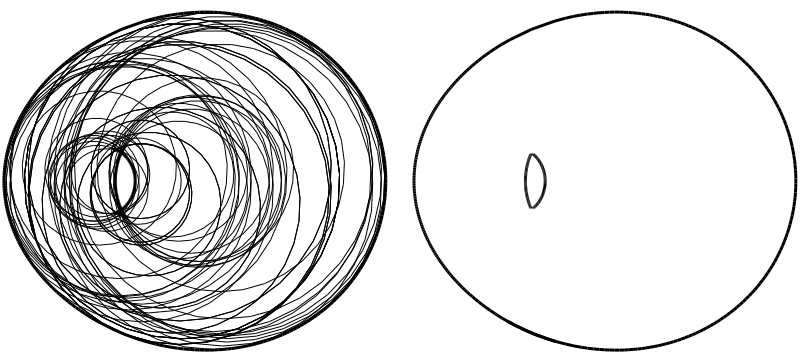

Figure 15. 2-parameter set of circles bounded by the untrimmed part of the cyclographic silhouette (left), trimmed silhouette (right)

is an irreducible algebraic surface. Figure 15 shows some circles in the corresponding 2-parameter set of circles. It illustrates that fact that the (trimmed) cyclographic silhouette appears as boundary of the domain covered by the set of circles.

Remark. Although it is not necessary for the computation of the silhouette, it is interesting to ask for the cyclographic contour of a quadric. The polarity $\kappa$ with respect to a quadric maps each point of the quadric to its tangent plane and vice versa. Hence, $\kappa$ maps the tangent planes of $\Gamma(\Phi)$ to the points of the contour $c(\Phi)$. By linearity of $\kappa$, it follows that the cyclographic contour of a quadric is in general an algebraic curve of order four (image of a developable surface of class 4$)$. It may be computed as the intersection of $\Phi$ with the polar image of $\Omega \subset \Gamma(\Phi)$, which is a quadratic cone. The same argumentation applies for arbitrary isophotes and thus we see the classical result that isophotes of quadrics are algebraic curves of order $\leq 4$.

\subsection{Three-parameter sets of circles}

These are the cyclographic images of solids in $\mathbb{R}^{3}$. Contributions to the boundary of domain $D(s)$ covered by the circle system can only be cyclographic images of boundary surfaces, edge curves and vertices of the solid.

Example 3.3. We discuss the tolerance region $D$ for a circle given by a fat midpoint $M$ and an interval $I=[r, R]$ for the radius ( $r$ shall be larger than the diameter of $M$ ). The set of all circles with a midpoint in the convex domain $M$ and a radius $\rho \in I$ has the following solid $S$ as cyclographic preimage: $S$ is bounded by the two planes $x_{3}=r, x_{3}=R$ and a cylinder surface with $x_{3}$-parallel rulings and base curve through the boundary curve $m$ of $M$. These three surfaces cannot have $\gamma$-planes as tangent planes and thus need not be considered for a contribution to the boundary of $D$. The contribution can only come from the two convex boundary curves $m_{r}$

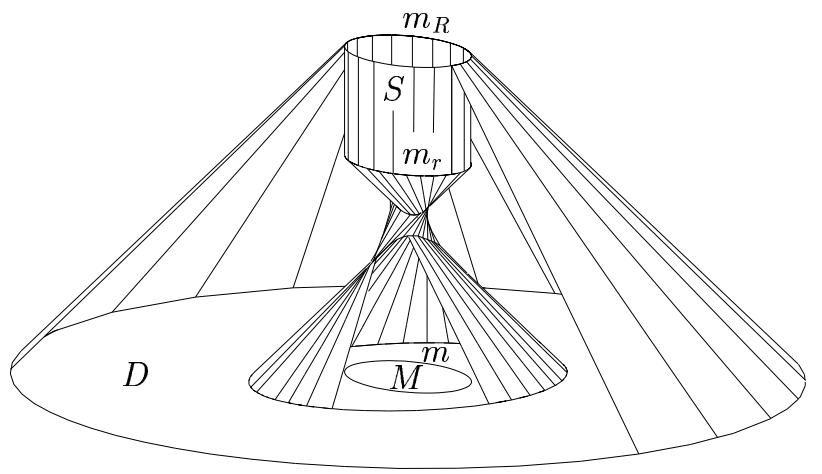

Figure 16. Cyclographic preimage of the tolerance region of a circle with fat center and radius $\rho \in[r, R]$

and $m_{R}$ which are translates of $m$ by vectors $(0,0, r)$ and $(0,0, R)$, respectively. The complete cyclographic image of $m_{r}$ is the two-sided offset of $m$ at distance $r$, and analogously the image of $m_{R}$ is the two-sided offset at distance $R$. The $\gamma$-developable through $m_{r}$, which leads to the 'exterior' offset of $m$ at distance $r$ (defined by $m+r n$ with outside unit normals $n$ of the curve $m$ ) cuts at points of $m_{r}$ locally into the solid; hence it does not contribute to $D$. The same holds for the interior offset of $m$ at distance $R$. The exterior offset of $m$ at distance $R$ is always convex. It bounds the outer boundary curve of the ring-shaped domain $D$. The inner boundary curve is the trimmed interior offset of $m$ at distance $r$. This simple result can be obtained easily without the cyclographic model (see also [16]). We used it here just to give an example of the concept. Figure 16 shows an axonometric view.

\section{Tolerance zones for circles in the plane}

\subsection{Circles through a fat point}

For a tolerance analysis of circle constructions with help of the cyclographic model, the following problem is of fundamental importance: Given a convex domain $K$ (fat point), find the cyclographic preimage $S$ of all circles through the fat point, i.e., circles intersecting $K$.

A circle intersecting $K$, which contributes to the boundary of $S$ must possess a circle in an arbitrarily small neighborhood of itself, which does not intersect $K$. Hence, such a circle must be tangent to $K$. All circles touching $K$ have as cyclographic preimage two $\gamma$-developables $\Gamma_{1}, \Gamma_{2}$ through the boundary $k$ of $K$. Confining to a positive radius, we may restrict to the part in $x_{3}>0$. Let $\Gamma_{1}$ belong to circles which are centered outside $K$ and touch $K$ from outside. Together with $E^{2}: x_{3}=0$, the surface $\Gamma_{1}$ confines an unbounded 

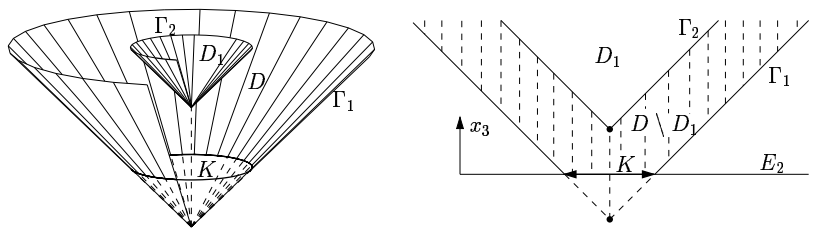

Figure 17. Cyclographic preimage of circles through a fat point (disk), axonometric and front view

convex domain $D$ in $\mathbb{R}^{3}$

Now, we have to remove that subset $D_{1}$ of $D$, whose cylographic image circles cover $K$. Here and in the following we say that a circle 'covers' a set $A$, if the closed circular disk contains $A$. The set $D_{1}$ is bounded by the part of $\Gamma_{2}$, whose cyclographic image circles touch $k$ and cover $K$. This part is therefore the one which is visible for parallel projection in direction $(0,0,-1)$. In general, it contains edges which are self-intersections of the complete surface $\Gamma_{2}$ (see Fig. 18). $D_{1}$ is convex: Given two points $a, b \in D_{1}$, their cyclographic image circles cover $K$. The images circles of the points $x$ on the straight line segment with end points $a, b$ cover the intersection of the circular disks bounded by $\zeta(a)$ and $\zeta(b)$. Hence, $\zeta(x)$ also covers $K$. Thus, $x \in D_{1}$, and we have proved the convexity of $D_{1}$.

Theorem 2. The cyclographic preimage of all circles (with positive radius) through a fat point $K$ is the difference set $D \backslash D_{1}$ of two unbounded convex domains in $\mathbb{R}^{3} . \quad D$ is bounded by the convex domain $K$ in the plane $E^{2}$ and part of a $\gamma$-developable $\Gamma_{1}$ through the boundary $k$ of $K$; its points are cyclographic preimages of circles touching $k$ from outside. $D_{1}$ is bounded by the part of the second $\gamma$-developable $\Gamma_{2}$ through $k$, which is visible for projection parallel to the vector $(0,0,-1)$. This boundary possesses cyclographic image circles, which touch $k$ and cover $K$.

Example 4.1. As the simplest example, let us consider a circular disc $K$ with center $\left(m_{1}, m_{2}\right)$ and radius $r$. The two $\gamma$-developables through the circle $k$ are two $\gamma$-cones $\Gamma_{1}$ (with vertex $\left.\left(m_{1}, m_{2},-r\right)\right)$ and $\Gamma_{2}$ (with vertex $\left.\left(m_{1}, m_{2}, r\right)\right) . D$ is bounded by $K$ and the (smooth) part of $\Gamma_{1}$ which lies above $E^{2} . D_{1}$ is bounded by the upper half of the cone $\Gamma_{2}$ (Fig. 17).

Example 4.2. Another example is illustrated in Figures 18 and 19. The fat point $K$ in $E^{2}$ is bounded by an ellipse $k$. The complete $\gamma$-developable through $k$ is an algebraic developable surface of class four (connection with the ideal conic $\Omega$ ). The surface has four conics as self-intersections: one is the ideal conic $\Omega$ and another one is the ellipse $k$. The remaining two self-intersections lie in $z$-parallel planes through the axes of the ellipse. In the upper halfspace $z \geq 0$,

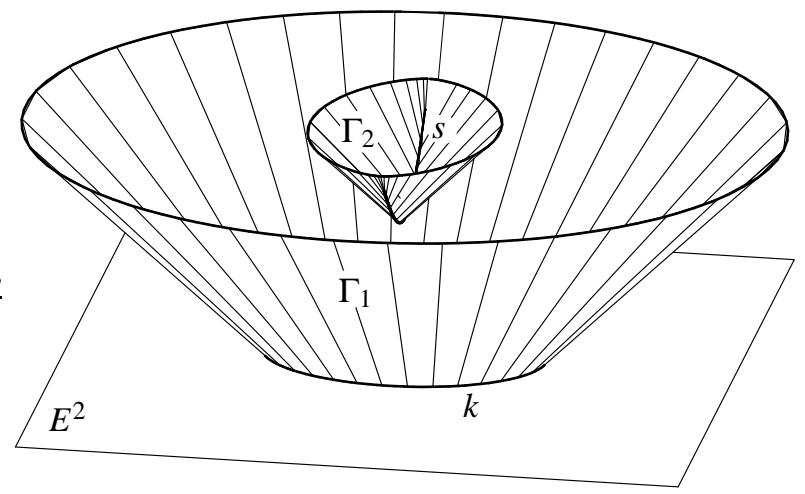

Figure 18. Boundary surfaces of cyclographic preimage of circles through a fat point (bounded by an ellipse $k$ )

the outer part $\Gamma_{1}$ is free of self intersections, but the other component $\Gamma_{2}$ has two conic arcs as self-intersection; one of them appears as edge of the boundary of $D_{1}$ (Fig. 19).

\section{Fat circle through three fat points}

As an application of the previous considerations, let us study the region $D$ covered by all circles that intersect three convex domains $K_{1}, K_{2}, K_{3}$. They shall be arranged such that they form a fat triangle. This requires that they may not be viewed as collinear, i.e., no line intersects all three domains $K_{i}$. In particular, this implies that the zones $K_{i}$ are pairwise disjoint.

The cyclographic preimage of this 'fat circumcircle' is the intersection $S$ of the domains $S\left(K_{i}\right)$, characterizing circles which intersect $K_{i}$ according to Theorem 2 . The bounding surfaces of $S$ are $\gamma$-developables only; hence their cyclographic silhouettes are the boundary curves $k_{i}$ of $K_{i}$. The edges occurring as intersections of two $\gamma$-developables also possess just parts of $k_{i}$ as cyclographic images. Hence, other contributions to the boundary of $D$ can only be parts of circles, namely the cyclographic images of the vertices of $S$. Not all of these vertices need to generate part of the boundary. One of the vertices, say $v_{---}$belongs to a circle, which covers all three $K_{i}$. Another one, $v_{+++}$has as cyclographic image a circle touching the $K_{i}$ from outside. It is not trivial to prove that these vertices (and image circles) exist. The straight line segment $v_{---} v_{+++}$generates circles all of which intersect $K_{i}, i=1,2,3$. These circles cover a domain which contains all $K_{i}$ and thus the boundaries $k_{i}$ of $K_{i}$ do not contribute to the boundary of $D$. Together with Theorem 2 on the boundary of $S\left(K_{i}\right)$ we can state the following result. For its formulation we further note: A circle touches a domain $K_{i}$ from outside or covers it iff it intersects $K_{i}$ in exactly one point. 


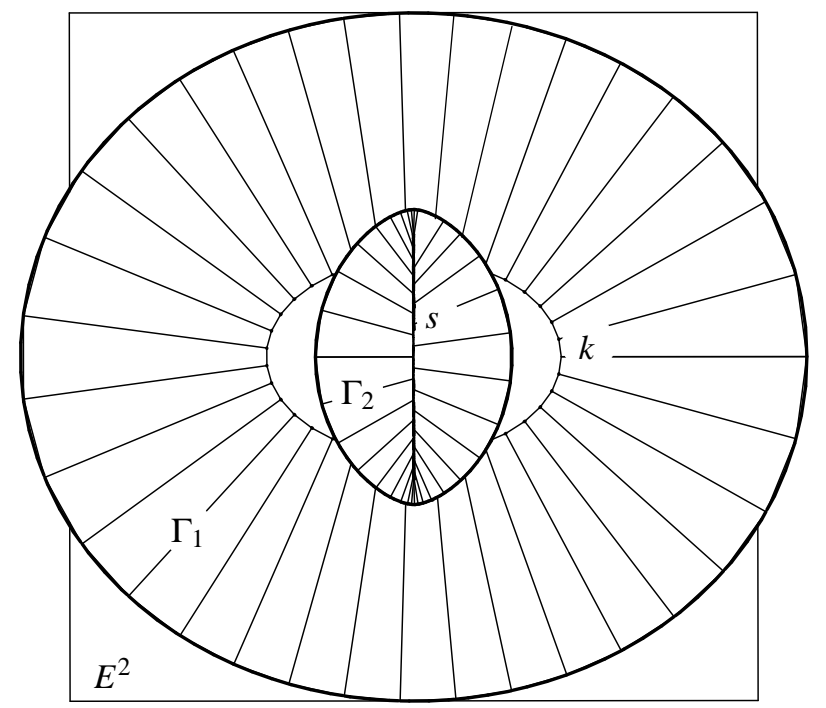

Figure 19. Top view of $\Gamma_{1}, \Gamma_{2}$, ellipse $k$ and self-intersection $s$ of $\Gamma_{2}$

Theorem 3. The domain covered by all circles through three fat vertices $K_{1}, K_{2}, K_{3}$ of a triangle is bounded by parts of circles which intersect $K_{i}$ in exactly one point.

In case of three circular disks $K_{i}$, there are 8 candidate circles, but two of them, namely circles touching all three $k_{i}$ from outside or covering all three (the cyclographic images of the vertices $v_{---}$and $v_{+++}$) do not contribute to the boundary [46] (see Fig.20). An analogous statement can be made about $\zeta\left(v_{---}\right)$and $\zeta\left(v_{+++}\right)$for general convex domains $K_{i}$.

For practical calculations it seems advantageous to work with piecewise linear and circular boundary curves $k_{i}$ of the fat points. Then the computation of the vertices of the solid $S$ in $\mathbb{R}^{3}$ can be done efficiently. It amounts only to intersecting $\gamma$-planes or $\gamma$-cones and thus needs no numerical approximation.

\subsection{Circles tangent to a toleranced line}

Consider a fat line spanned by two fat points $K_{1}, K_{2}$. The fat line consists of all lines intersecting both $K_{1}$ and $K_{2}$. This set of lines is a set of points in dual projective plane and thus we can speak of its boundary $B$. Dual to the boundary of the intersection of two domains (2-parameter point sets) in the plane, we see that $B$ consist of all lines which touch at least one of the convex domains $K_{i}$ and intersects the other one. If the boundaries of $K_{i}$ are smooth, $B$ is piecewise smooth as a set of lines: its envelope consists of four segments $c_{11}, c_{12}, c_{21}, c_{22}$ on the boundary curves of $K_{i}$ (between the contact points of common tangents). Disconti-

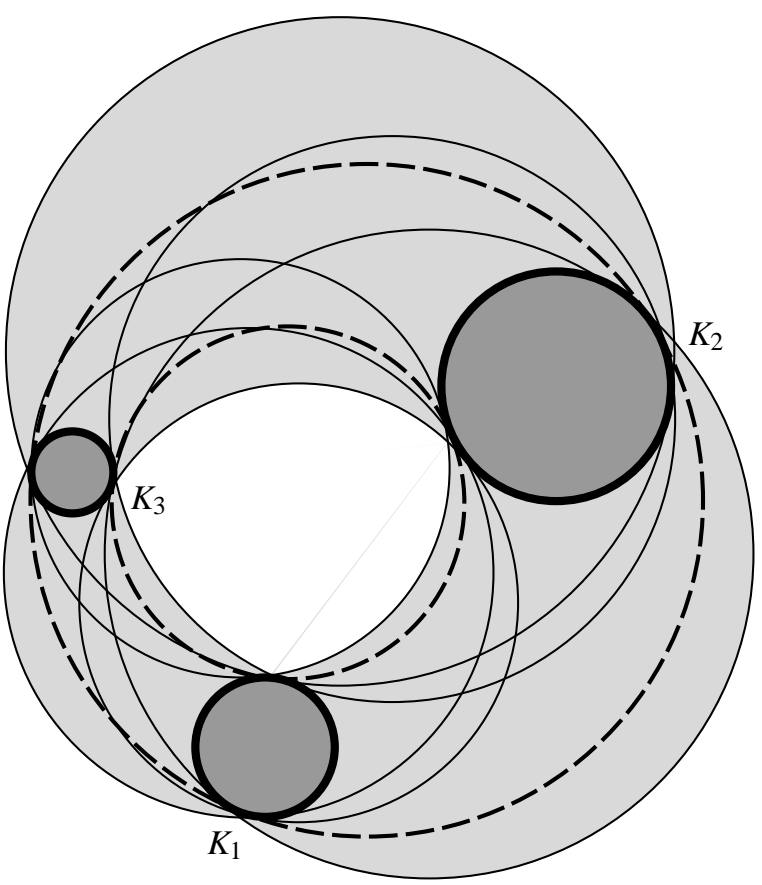

Figure 20. Fat circle through three fat points

nuities of the contact point with the envelope appear at the common tangents (see Fig. 21).

We now look at the cyclographic preimage of the set of circles which touch the fat line from a given side. All circles touching an oriented line $L$ have their cyclographic preimages in a $\gamma$-plane through $L$. Since we are confining to a positive radius, we need just the half-plane in $x_{3}>0$. In this way, we associate a $\gamma$-plane with each line in the fat line. The domain $S$ traced out in space is bounded by parts of the $\gamma$-planes to the common tangents of $K_{1}, K_{2}$ and parts of the $\gamma$-developable surfaces through the curve segments $c_{i j}$.

To study the domain $S$, we may intersect it with a plane $\varepsilon: x_{3}=r=$ const. It belongs to circles with radius $r$. The orthogonal projection of such a horizontal slice $\varepsilon \cap S$ into $E^{2}$ is the tolerance region for the centers of circles with radius $r$ which touch the fat line.

For $r=0$, we get the boundary of the tolerance zone $S \cap E^{2}$ to the fat line; here, the curves $c_{i j}$ do not contribute to the boundary. However, increasing $r$, we see that the mentioned developable surfaces contribute to the boundaries of the slices; these curved boundary parts are of course offsets of $c_{i j}$ at distance $r$ (Fig. 21).

As an application we may discuss the fat circle touching three given fat lines. Each fat line may be given as connection of two fat points; thus, altogether we admit 6 input points (not just a triangle). We have to intersect the three domains $S_{1}, S_{2}, S_{3}$ corresponding to the contacting circles 


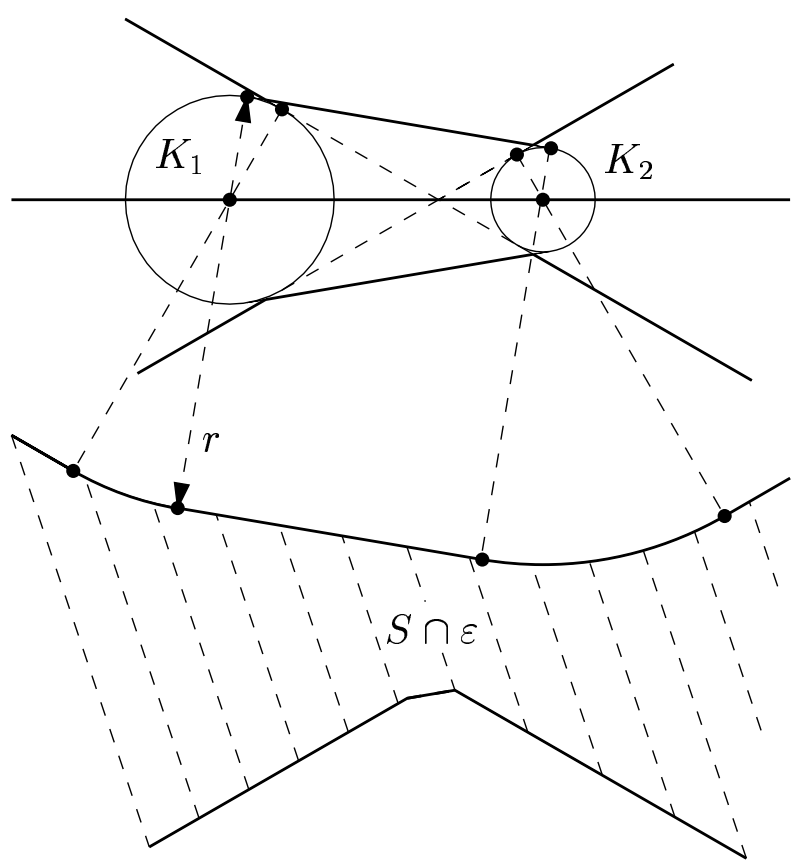

Figure 21. Tolerance domain for the centers of circles with fixed radius $r$ which are tangent to a toleranced line

of each of the three lines. The intersecting solid $S$ has as its boundary surfaces only $\gamma$-planes and $\gamma$-developable surfaces. Hence, there will be no nontrivial cyclographic contours and silhouettes. This proves the following result:

Theorem 4. Let us consider three fat lines $L_{A}, L_{B}, L_{C}$, each of them given as connection of two fat points $\left[A_{1}, A_{2}\right]$, $\left[B_{1}, B_{2}\right]$ and $\left[C_{1}, C_{2}\right]$, respectively. Then, the domain covered by all circles which are tangent to the three fat lines at a given side is bounded by parts of circles which touch triples of lines $H_{A}, H_{B}, H_{C}$ of the following type: $H_{A}$ touches either $A_{1}$ or $A_{2}$ (at a boundary point whose tangent intersects $A_{2}$ or $A_{1}$, resp.) or a common tangent of $A_{1}, A_{2}$; analogous statements hold for $H_{B}, H_{C}$.

Further research will be necessary to make a sharper statement and to rule out parts of $A_{i}, B_{i}, C_{i}$ on the boundary.

With an appropriate definition of the side from which the circle shall touch the three given lines, the resulting tolerance zone is the tolerance zone for the inscribed circle of a fat triangle. Its vertices are the intersections of the tolerance zones to the given lines. However, given three fat points $A, B, C$, defining the three fat connecting lines, and then doing the present construction does not give the tolerance region for the inscribed circle to the triangles with the given fat vertices $A, B, C$. This is so, because the tolerance

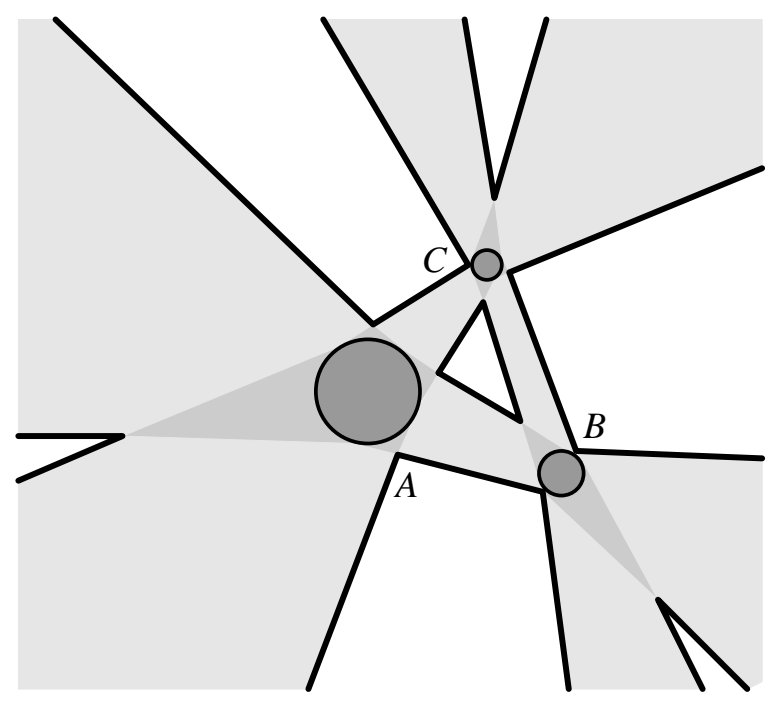

Figure 22. Fat triangle

regions to the edges of the fat triangle have intersections which cover the given fat points $A, B, C$, but they are in general larger than those (see Fig. 22).

\subsection{Tangents to a fat circle}

Let us consider a fat circle represented in the cyclographic model by some solid $S \in \mathbb{R}^{3}$. We would like to pass tangents through a given point in the plane to the circles in this 3-parameter family and ask for the tolerance zones of the contact points.

To start with a simple case, we look at tangents parallel to a given (at the moment) precise line $L \in E^{2}$ with a unit normal vector $\left(n_{1}, n_{2}\right)$. The two lines, parallel to $L$, which touch a circle $c$ with center $\left(p_{1}, p_{2}\right)$ and radius $p_{3}$ have contact points $\left(p_{1}, p_{2}\right) \pm p_{3}\left(n_{1}, n_{2}\right)$. These points may be viewed as images of the cyclographic preimage $p=\left(p_{1}, p_{2}, p_{3}\right)$ of $c$ under a projection parallel to vectors $l_{1}=\left(n_{1}, n_{2}, 1\right)$ or $l_{2}=\left(n_{1}, n_{2},-1\right)$. The projection lines are $\gamma$-lines in planes orthogonal to $L$. We see that in the present situation the tolerance zones for the contact points are the images of the cyclographic preimage $S$ under projections parallel to vectors $l_{1}$ and $l_{2}$.

This is extended to the case of an inprecise direction of $L$. Instead of $l_{1}$, we have the rulings of a part $\Gamma_{1}$ of a $\gamma$ cone as possible projection rays, and analogously for $l_{2}$. Let $a_{1}, b_{1}$ be the end rulings of the cone segment $\Gamma_{1}$. Now, the tolerance zone for one of the contact points contains at its boundary the following curves: parts of the boundaries of the projections of $S$ parallel to $a_{1}, b_{1}$ and part of the cyclographic image of $S$. For the latter, only those cyclographic contour points on $S$ are admitted at which a normal of $S$ is parallel to a normal of the cone segment $\Gamma_{1}$, i.e., a tangent 


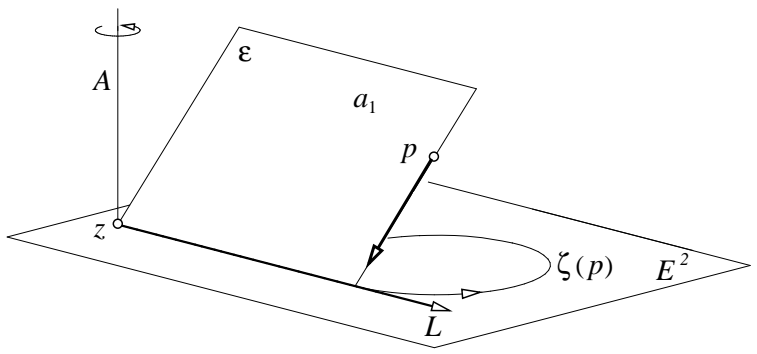

Figure 23. Construction of tangents to a fat circle

of the cyclographic image is within the given range of directions of $L$.

Contact points of tangents through a given (precise) point $z \in E^{2}$ can also be successfully discussed with help of the cyclographic model. Consider an oriented line $L$ through $z$. Then, the cyclographic preimage of the circles touching $L$ are points $p$ in a $\gamma$-plane $\varepsilon$ through $L$. The corresponding contact points are obtained as projections of $p$ with $\gamma$-lines in the plane $\varepsilon$ (Fig. 23). Rotating the pencil of $\gamma$-lines in $\varepsilon$ around the $x_{3}$-parallel line $A$ through $z$, we obtain a two-parameter set of lines, i.e. a line congruence $C$. We call it rotationally symmetric $\gamma$-congruence with axis $A$, see Figure 24. In line geometric terms, $C$ is an algebraic congruence with bundle degree two (algebraically counted, two lines of $C$ pass through a general point) and field degree two (2 lines of $K$ lie in a general plane).

The two lines of $C$ through a given point $p \in \mathbb{R}^{3}$ intersect $E^{2}$ in the contact points of those tangents of $\zeta(p)$, which pass through $p$. Hence, the contact regions are again obtained via a projection, but now the projecting lines are taken from the congruence $C$. The boundary of the projection of the solid $S$, representing the fat circle, is formed by image curves of edges and silhouettes of surfaces, arising as intersections of those lines $l \in C$, which are tangent to $S$. We see that the nontrivial part of the boundary again amounts to the determination of the silhouette with respect to some projection. Let us summarize:

Theorem 5. The tolerance regions of the contact points of tangents from a fixed point $z$ to the circles of a k-parameter family are obtained as follows: Project the cyclographic preimage of the circle set via the rotationally symmetric congruence of $\gamma$-lines with axis $A \ni z$ into $E^{2}$.

This generalized projection deserves further studies, both from an algebraic and differential geometric viewpoint. We also mention that the tangents from a fat point can be discussed in a similar way. However, at each point

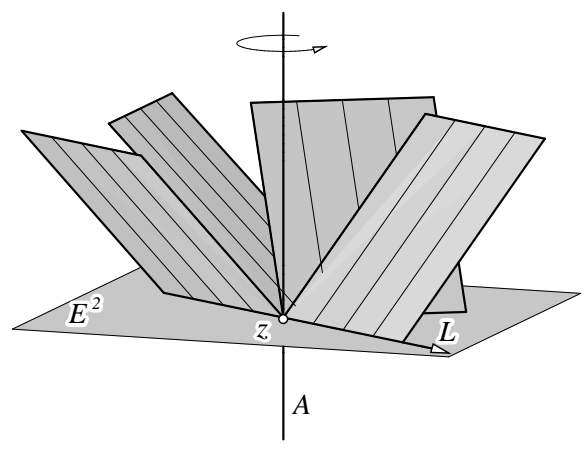

\section{Figure 24. Rotationally symmetric $\gamma$ - congruence}

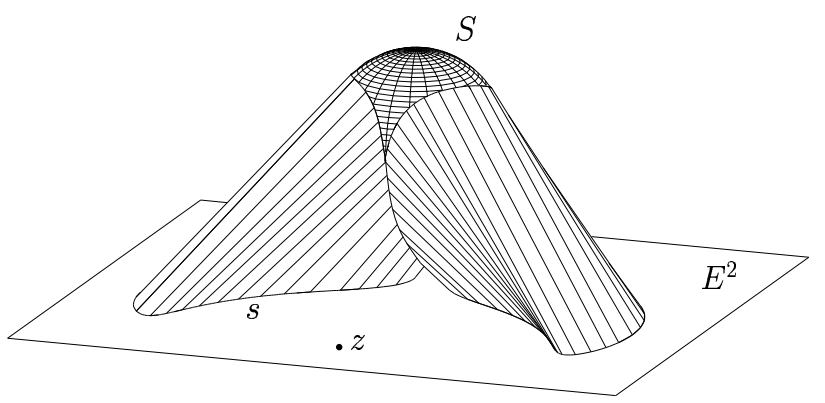

Figure 25. Cyclographic preimage $S$ of fat circle and construction of tolerance region of contact points of tangents through $z$ to the fat circle

in space, we then have two segments of $\gamma$-cones for the projection.

Aspects of the computation of contours of surfaces with respect to line congruences have been studied by Elber and Cohen [9] in connection with accessibility in NC machining.

Example 4.3. Consider a fat circle, whose cyclographic preimage is a sphere $S$. In the plane $E^{2}$, the fat circle consists of a two-parameter set of circles. We construct the tolerance zones for the contact points of tangents from a fixed point $z$ to these circles according to Theorem 5. Fig. 25 shows the two components of the ruled surface formed by those lines in the rotationally symmetric $\gamma$-congruence, which touch the sphere $S$. The intersection with $E^{2}$ yields the boundaries of the tolerance zones for the two contact points, see Figure 26. Whereas a parallel projection maps a convex surface onto a convex silhouette, the present generalized projection obviously does not possess this convexity preserving property. 


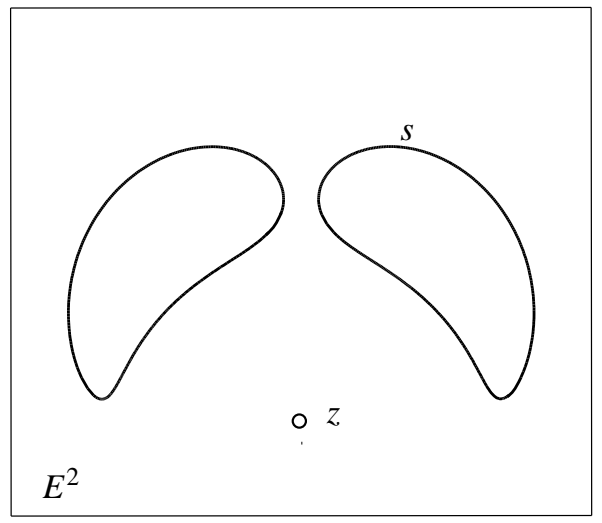

\section{Figure 26. Tolerance region of contact points of tangents through $z$ to a fat circle}

\section{Conclusion and future research}

We have outlined geometric concepts for a worst case tolerance analysis of CAD constructions. From a theoretical viewpoint, linear constructions as well as linear freeform curve schemes are understood very well.

We have also shown how the cyclographic mapping helps to study planar constructions involving circles. Here, further research is needed to get a better understanding of the global behaviour of the resulting tolerance zones.

Missing planar constructions concern normals to lines and reflections in the plane, which will be subject of a separate contribution. Very much remains to be done in three dimensions, both concerning metric constructions and reliable approximations of tolerance zone boundaries of surfaces.

Another important issue which needs to be addressed in the future is an efficient implementation. For that, it will be necessary to make limitations on the shapes which are used to represent the tolerance zone boundaries.

\section{Acknowledgements}

This research has been supported in part by projects P12252-MAT and P13648-MAT of the Austrian Science Fund. R. Ait-Haddou acknowledges support by a fellowship from Vienna University of Technology and by the Geomechanics Project at the University of Calgary. We would also like to thank N. Pomaroli for his help with some figures.

\section{References}

[1] S. Abrams, W. Cho, C.-Y. Hu, T. Maekawa, N. Patrikalakis, E. Sherbrooke, and X. Ye. Effi cient and reliable methods for rounded-interval arithmetic. Computer-Aided Design, 30:657-665, 1998.

[2] M. Aichinger. A collision problem for worst case tolerance objects. PhD thesis, Kepler University Linz, 1996.

[3] R. Ait-Haddou, L. Biard, and M. A. Slawinsky. Pythagorean hodograph curves in the Minkowski plane I: geometric characterization. Submitted to Computer Aided Geometric Design.

[4] R. Ait-Haddou, L. Biard, and M. A. Slawinsky. Pythagorean hodograph curves in the Minkowski plane II: dual B'ezier characterization. Submitted to Computer Aided Geometric Design.

[5] W. Blaschke. Untersuchungen über die Geometrie der Speere in der Euklidischen Ebene. Monatshefte für Mathematik und Physik, 21:3-60, 1910.

[6] J. Coolidge. A Treatise on the Circle and the Sphere. Clarendon Press, Oxford, 1916.

[7] B. Cousinery. G'eom étrie Perspective. Paris, 1828.

[8] C. de Boor, K. Höllig, and M. Sabin. High accuracy geometric Hermite interpolation. Computer Aided Geometric Design, 4:269-278, 1987.

[9] G. Elber and E. Cohen. A unifi ed approach to accessibility in 5-axis freeform milling environments. In B. C. G.J. Olling and R. Jerard, editors, Machining impossible shapes, pages 33-41, Boston, 1998. Kluwer.

[10] R. Farouki. Pythagorean-hodograph curves in practical use. In R. E. Barnhill, editor, Geometry Processing for Design and Manufacturing, pages 3-33, Philadelphia, 1992. SIAM.

[11] R. Farouki and T. Goodman. On the optimal stability of the Bernstein basis. Mathematics of Computation, 216:15531566, 1996.

[12] R. Farouki, H. Moon, and B. Ravani. Algorithms for Minkowski products and implicitly-defi ned complex sets. Preprint, 1999.

[13] R. Farouki, H. Moon, and B. Ravani. Minkowski geometric algebra of complex sets. Geometriae Dedicata, 2000.

[14] R. Farouki and T. Sakkalis. Pythagorean hodographs. IBM J. Res. Develop., 34:736-752, 1990.

[15] P. Gruber and J. Wills. Handbook of Convex Geometry, volume 1,2. North Holland, Amsterdam, 1993.

[16] C. U. Hinze. A contribution to optimal tolerancing in 2dimensional computer aided design. $\mathrm{PhD}$ thesis, Kepler University Linz, 1994.

[17] C. Hu, T. Maekawa, E. Sherbrooke, and N. Patrikalakis. Approximation of measured data with interval B-splines. Computer-Aided Design, 29:791-799, 1997.

[18] C.-Y. Hu, N. Patrikalakis, and X. Ye. Robust interval solid modeling: part I, representations. Computer-Aided Design, 28:807-817, 1996.

[19] C.-Y. Hu, N. Patrikalakis, and X. Ye. Robust interval solid modeling: part II, boundary evaluation. Computer-Aided Design, 28:819-830, 1996.

[20] C.-Y. Hu, N. Patrikalakis, and X. Ye. Robust interval algorithm for surface intersections. Computer-Aided Design, 29:617-627, 1997.

[21] A. Kaul and R. Farouki. Computing Minkowski sums of plane curves. Intl. J. Computational Geometry \& Applications, 5:413-432, 1995. 
[22] R. Krasauskas and C. Mäurer. Studying cyclides with Laguerre geometry. Comput. Aided Geom. Design, 17(2):101$126,2000$.

[23] I. Lee, M. Kim, and G. Elber. Polynomial/rational approximation of Minkowski sum boundary curves. Graphical Models and Image Processing, 60:136-165, 1998.

[24] S. Leopoldseder. Cone spline surfaces and spatial arc splines. PhD thesis, Vienna Univ. of Technology, 1998.

[25] S. Leopoldseder and H. Pottmann. Approximation of developable surfaces with cone spline surfaces. Computer-Aided Design, 30:571-582, 1998.

[26] Q. Lin and J. Rokne. Disk B 'ezier curves. Comp. Aided Geometric Design, 15:721-737, 1998.

[27] T. Maekawa. An overview of offset curves and surfaces. Computer Aided Design, 31:165-173, 1999.

[28] D. S. Meek and D. J. Walton. Planar osculating arc splines. Comp. Aided Geometric Design, 13:653-671, 1996.

[29] H. Moon. Minkowski Pythagorean hodographs. Comp. Aided Geometric Design, 16:739-753, 1999.

[30] R. Moore. Interval Analysis. Prentice-Hall, Englewood Cliffs, NJ, 1966.

[31] E. Müller and J. Krames. Vorlesungen über Darstellende Geometrie II: Die Zyklographie, volume 2. Deuticke, Wien/Leipzig, 1929.

[32] M. Peternell and H. Pottmann. Computing rational parametrizations of canal surfaces. Journal of Symbolic Computation, 23:255-266, 1997.

[33] H. Pottmann. Rational curves and surfaces with rational offsets. Comp. Aided Geometric Design, 12:175-192, 1995.

[34] H. Pottmann. General offset surfaces. Neural, Parallel \& Scientifi c Comp., 5:55-80, 1997.

[35] H. Pottmann and G. Farin. Developable rational B'ezier and B-spline surfaces. Computer Aided Geometric Design, 12:513-531, 1995

[36] H. Pottmann and M. Peternell. Applications of Laguerre geometry in CAGD. Comp. Aided Geometric Design, 15:165186, 1998.

[37] H. Pottmann and J. Wallner. Approximation algorithms for developable surfaces. Comp. Aided Geometric Design, 16:539-556, 1999.

[38] A. A. G. Requicha. Towards a theory of geometric tolerancing. Int. J. of Robotics Research, 2:45-60, 1983.

[39] H. Schaal. Zur Konstruktion der Krümmungskreise des scheinbaren Umrisses einer Fläche bei Zentral- und Parallelprojektion. Sitzungsberichte d. Bayrischen Akademie d. Wissenschaften, math.-naturwiss. Klasse, 17:277-310, 1960.

[40] T. Sederberg and R. Farouki. Approximation by interval B’ezier curves. IEEE Computer Graphics, 12:87-95, 1992.

[41] G. Shen and N. Patrikalakis. Numerical and geometric properties of interval B-splines. Int. J. Shape Modeling, 4:31-62, 1998.

[42] S. Tuohy, T. Maekawa, G. Shen, and N. Patrikalakis. Representation of geophysical maps with uncertainty. In N. M. Thalmann and D. Thalmann, editors, Communicating with Virtual Worlds, volume 4 of Proc. of CG International '93, Tokyo, 1993. Springer.

[43] S. T. Tuohy and N. M. Patrikalakis. Nonlinear data representation for ocean exploration and visualization. Journal of Visualization and Computer Animation, 7:125-139, 1996.
[44] J. U. Turner. Tolerances in computer-aided geometric design. PhD thesis, Rensselaer Polytechnic Institute, Troy, NY, 1987.

[45] H. Vogler. Ein einfacher Beweis eines Satzes von H. Schaal über den Krümmungsmittelpunkt des scheinbaren Umrisses einer Fläche. Elemente d. Math., 17:79-81, 1962.

[46] J. Wallner, R. Krasauskas, and H. Pottmann. Error propagation in geometric constructions. Computer-Aided Design, to appear, 2000.

[47] J. Wallner, T. Sakkalis, T. Maekawa, H. Pottmann, and G. Yu. Self-intersections of offset curves and surfaces. Preprint, 1999. 